\title{
Effective uterovaginal anastomosis done in cervical agenesis with menouria R Sultana ${ }^{1}$, N Haque ${ }^{2}$, F Begum ${ }^{3}$
}

\begin{abstract}
Agenesis of the uterine cervix is uncommon uterine anomaly. We report a case of 22 year old woman who was admitted in a private hospital with menouria and pelvic mass. Examination found no communication between vagina and uterus. Cervix was absent. Utero-vaginal anastomosis was done and a Foleys catheter was inserted in the uterine cavity for 14 days. During follow up of 1 month and 6 month after surgery patient was menstruating normally and examination revealed that the communication between the uterus and vagina was healed and had been sexually active at 6 months after the procedure. So uterovaginal anastomosis can be safely done in cervical agenesis.
\end{abstract}

Bang Med J Khulna 2013; 46 : 28-30

\section{Introduction}

The incidence of Mullarian hypoplasia / agenesisType I anomalies is about $0.1 \%$ of anomalies in general population and about $3 \%$ of all uterine anomalies. 1 Congenital cervical agenesis is a rare anomaly of the female genital tract. In addressing the problems there have been applied different conservative surgical techniques; cervical canalization, cervical reconstruction and uterovaginal anastomosis. Conservative surgical treatment of women with these anomalies has remained controversial. ${ }^{2}$ Total hysterectomy is still being suggested as an option in their treatment in case of conservative surgical failure. ${ }^{3}$ Congenital absence of the cervix is a rare condition and occurs in 1 in 80,000 to 100,000 births. 4 It is known to be associated with both partial and complete vaginal aplasia and with renal anomalies. According to the American Fertility Society, cervical agenesis or dysgenesis should be classified as type IB Mullerial anomaly. ${ }^{5}$ Cervical agenesis involves a complete lack of any substantial cervical tissue where the lower uterine segment narrows to terminate in a peritoneal sleeve at a point well above the normal communication with the vaginal apex. Cervical dysgenesis in which varying portions of the cervical body develop may also occur. Structural defects of the uterus and vagina are associated with a host of gynecological and obstetric clinical problems. The defects are caused by either a genetic error or exposure to a teratogen during embryonic development. Presentation is usually with primary amenorrhoea and cyclical lower abdominal pain. In a recent retrospective view of 18 patients $39 \%$ had associated vaginal aplasia. Endometriosis or pelvic infection may result from the chronic hematometra. The presence of normal uterine corpus is a challenge for the clinician because a successful repair could restore normal menses and potentially preserve the patients fertility. The most available successful surgical methods employed to date for cervical agenesis have involved a transvaginal or transabdominal approach to create an ostium through the dense fibrous cavity with uterus and vagina by application of stents. ${ }^{6}$

\section{Case Report}

A 22 year old young lady referred to Islamic bank hospital with the complaints of pelvic mass, menouria and pelvic pain. On examination she had normal secondary sexual characters. There was no significant surgical or medical history. All routine blood test and hormone profiles were normal. A mass about 16 weeks size felt in lower abdomen, pervaginal examination showed the normal length of vagina, uterus about 14 weeks size, but no cervical tissue felt. There appeared to be no communication between the proximal vagina and the lower uterus. She was duly counseled and admitted for surgery in Islamic bank hospital Khulna on 2nd July 2012 to connect the uterus to the proximal vagina. Initially cystoscopy was done by Urologist and findings

1. Razia Sultana FCPS, Consultant Obs \& Gynae, Fultala Health Complex, Khulna

2. Nazmul Haque MS, Consultant Urologist, Sheikh Naser Hospital, Khulna

3. Ferdousi Begum DGO. Medical Officer, Khulna Medical College Hospital 
Bang Med J Khulna 2013; 46

were external urethral orifice, internal orifice and trigone of bladder was normal. There was a
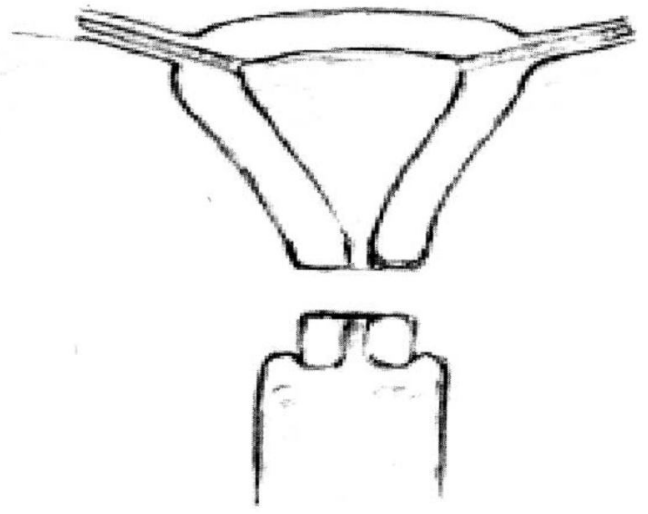

Figure-1. Diagramamtic representation of cervical dysgenesis showing cervical fragmentation

fistulous opening in right lateral wall of bladder through which a guide was tried to introduce but it cannot be done. According to the view of Urologist the vesicouterine fistula was very small and as we have made a uterovaginal outflow for menstrual period so fistula was not needed to be repaired. On vaginoscopy no cervical tissue and canal was found. Reconstructive surgery was planned by transabdominal and transperineal approach. Cervical agenesis was diagnosed

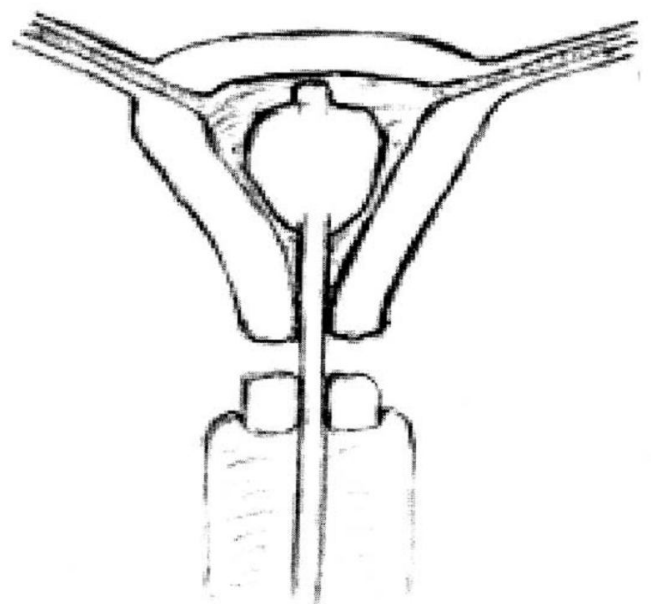

Figure-2. Insertion of Foleys catheter in the uterine cavity.

clinically and by intraoperative findings. Uterus was enlarged and right sided ovary was healthy. On left side there was a large chocolate cyst which was enucleated and uterus up to internal os present but there was no cervical remnant. So surgical diagnosis was cervical agenesis, vesico uterine fistula, haematometra, chocolate cyst of left ovary and endometriosis peritonei. Utero- vaginal anastomosis done by giving lower vertical incision in the lower part of the body of the uterus, dilator of $4 \mathrm{~mm}$ was introduced through endometrial cavity but it was reached up to the isthmic part of uterus which end in blind pouch. Then a 16 FR Foleys catheter is introduced in the uterine cavity after giving an incision in vaginal pouch and uterovaginal junction is closed by $2 / 0$ vicryl and uterine cavity was closed by interrupted sutures. Photograph could not be taken but schematic representation (Fig. 1-3) were done for understanding of operative procedure.

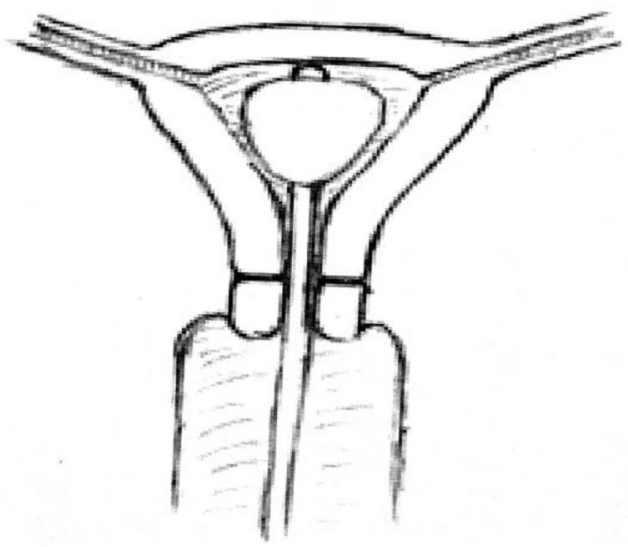

Figure-3. Cervico-cervix anastomosis

Operation performed were removal of chocolate cyst, resection of fibrous tissue around supravaginal uteri, anastomosis of isthmico uterovaginalis and drainage of pouch of douglas. Patient recovered well and discharged on 7 th postoperative day. Postoperatively she received antibiotic, oral contraceptive and vaginal douches. Postoperative follow up at 1 month and 6 month showed that she menstruate normally and she is also sexually active without difficulty.

\section{Discussion}

In the general female population, genital malformations occur with an incidence of 1-5\%. Jacobet et al 1989 made an extensive review of the published cases of surgical reconstruction for correction of atretric cervix. Presence of patent tubes, a functioning endometrium with an outflow obstruction is associated with a high incidence (up to $77 \%$ ) of endometriosis. ${ }^{6}$ Abnormal development in one of the urinary or the reproductive system is a strong evidence to search congenital fusion of the cervical spine, short neck and limited range of motion in the cervical spine. The MURCS association, mullerian, renal, cervicothoracic somite dysplasia has also been associated with MRKH syndrome.7 Previously the recommended treatment of cervical agenesis was hysterectomy 
because complications of recanalizing the cervix were common and the possibility of a viable pregnancy was unlikely. ${ }^{8,9}$ Recent advances in reproductive technology and laparoscopic surgical techniques mean that conservative surgery is possibility and should be considered as the first line treatment option. 10

The largest study that examined outcomes in eighteen patients after open uterovaginal anastomosis found that $22 \%$ of patients required further surgery after the initial procedure. Furthermore those investigators reported six spontaneous pregnancies in four of these patients. Mullarian malformation is associated in 50\% of the cases with vaginal aplasia Any abdominal or pelvic acute or chronic pain in a pubescent girl must evoke an obstructive genital syndrome. Clinical examination easily eliminates hymenal imperforation or blind hemivagina, but might not differentiate cervical atresia from high vaginal diaphragm. Transabdominal or transperineal ultrasonography may specify the level of obstacle but seems not very reliable for the diagnosis of uterine cervix atresia. Vesicouterine fistula is rare accounting nearly $4 \%$ of all urogenital fistula. It may be congenital which is very rare or acquired after caesarean delivery through lower uterine segment is the main predisposing event. Menouria is rare event in gynecology, occurs following vesicouterine fistula (VUF) and one should always keep this possibility in mind when here is cyclical haematuria.

The management protocol for VUF should be individualized taking into consideration the etiology size, time interval between its occurrence and institution of treatment regimen. Magnetic Resonance Imaging (MRI) currently appears to be most reliable morphological examination for the diagnosis of uterovaginal malformations with a surgical correlation more than $80 \%$. Some rare cases of successful pregnancies have been reported after conservative surgical management. Some important facts regarding the surgical therapy of the cervical agenesis have been emerged as follows- the successful establishment of patent cervical canal has been possible in some cases. Partial or complete cervical agenesis is often but not always associated with partial or complete vaginal agenesis. Two reported deaths due to transgenital infection with secondary peritonitis should permit great attention to postoperative infection control. Cervical agenesis or dysgenesis is an extremely rare congenital anomaly. Conservative surgical approach to these patients involves uterovaginal anastomosis, cervical canalization and cervical reconstruction.

\section{References}

1. Grimbizis GF, Camus M, Tarlatzis BC, BontisJN, Devroey P. Clinical implications of uterine malformations and hysteroscopic treatment results ; Hum Reprod Update 2001; 7: 161-174.

2. Deffarges JV, Haddod B, Musset R, Paniel J. Uterovaginal anastomosis in women with uterine cervical atresia. Long term follow up and reproductive performance. A study of 18 cases. Hum Reprod 2001; 16 : 1722-5.

3. Rock ja, Carpenter SE, Wheeless CR, Jones HWJ. The clinical management of maldevelopment of uterine cervix. J Pelv Surg 1995; 1: 129 - 33.

4. Creighton SM, Daves MC; Cutner A. Laparoscopic management of cervical agenesis Fertil Steril. 2006; 85 ; $1510,13-15$.

5. The American Fertility Society classification of adnexal adhesion, distal tubal occlusion secondary to tubal ligation, tubal pregnancies, Mullerian anomalies and intrauterine adhesions, Fertil Steril 1988; 49: 944-55.

6. Shoupe D.Uterovaginal anomalies: Management In Mishell DR, Goodwin TM, Brenner PT, (eds), Management of common problems in obstetrics and gynecology 4th edition, Massachusetts, Black well Publishing; 2002: 400-405.

7. LynosJK. MURCS association: mullerian duct, renal and cervical vertebral defects. In : Smith Recognizable patterns of human malformation , 5th ed. Philadelphia, Pa, WB Saunders; 1997: 666.

8. Hampton Ht, Role of the gynecologic surgeon in the management of Urogenital anomalies in adolescents Curopin Obstet Gynaecol 1990; 2: 812-8

9. Grinbizis GT,Tsalikis T, Mikos T, Papadopoulos n, Tarlatzis BC, Bontin JM. Successful end-to-end cervico-cervical anastomosis in a patient with congenital cervical fragmentation; a case report. Hum Reprod 2004; 19: 1204-10.

10. Deffanges JV, Haddad B, Muset R, Paniel BJ; Uterovaginal anastomosis in women with uterine cervix atresia; Long term follow up and reproductive performance. A study of 18 cases .Hum Reprod 2001; 16: 1722-5. 